\title{
The Association among Antioxidant Enzymes, Autoantibodies, and Disease Severity Score in Systemic Lupus Erythematosus: Comparison of Neuropsychiatric and Nonneuropsychiatric Groups
}

\author{
Yu-Jih Su, ${ }^{1,2}$ Tien-Tsai Cheng, ${ }^{1}$ Chung-Jen Chen, ${ }^{1}$ Wen-Chan Chiu, ${ }^{1}$ \\ Wen-Neng Chang, ${ }^{3}$ Nai-Wen Tsai, ${ }^{3}$ Chia-Te Kung, ${ }^{4}$ Wei-Che Lin, \\ Chih-Cheng Huang, ${ }^{3}$ Ya-Ting Chang, ${ }^{2,3}$ Chih-Min Su, ${ }^{2,4}$ Yi-Fang Chiang, \\ Ben-Chung Cheng, ${ }^{1,2}$ and Cheng-Hsien $\mathrm{Lu}^{2,3}$ \\ ${ }^{1}$ Department of Internal Medicine, Kaohsiung Chang Gung Memorial Hospital and Chang Gung University College of Medicine, \\ Kaohsiung 833, Taiwan \\ ${ }^{2}$ Department of Biological Science, National Sun Yat-Sen University, Kaohsiung 80424, Taiwan \\ ${ }^{3}$ Department of Neurology, Kaohsiung Chang Gung Memorial Hospital and Chang Gung University College of Medicine, \\ Kaohsiung 833, Taiwan \\ ${ }^{4}$ Department of Emergency Medicine, Kaohsiung Chang Gung Memorial Hospital and Chang Gung University College of Medicine, \\ Kaohsiung 833, Taiwan \\ ${ }^{5}$ Department of Radiology, Kaohsiung Chang Gung Memorial Hospital and Chang Gung University College of Medicine, \\ Kaohsiung 833, Taiwan \\ Correspondence should be addressed to Cheng-Hsien Lu; chlu99@ms44.url.com.tw
}

Received 25 February 2014; Accepted 1 April 2014; Published 27 April 2014

Academic Editor: Hung-Chen Wang

Copyright (C) $2014 \mathrm{Yu}$-Jih Su et al. This is an open access article distributed under the Creative Commons Attribution License, which permits unrestricted use, distribution, and reproduction in any medium, provided the original work is properly cited.

Background. Antioxidative capacity plays an important role in the severity of systemic lupus erythematosus (SLE), which is characterized by autoantibodies. This study aimed to determine the relationship among autoantibody titers, antioxidative stress reserve, and severity of SLE. Methods. The autoantibody titers, clinical markers, antioxidant enzyme levels, and disease activity index (SLEDAI-2k) of 32 SLE patients and 16 healthy controls were compared. We also compared both the neuropsychiatric (NPSLE) and nonneuropsychiatric (non-NPSLE) groups. Results. Superoxide dismutase in red blood cells was significantly lower in the SLE than in the control group. CRP levels are significant higher in SLE patients than in control group $(P=0.034)$. Among the autoantibodies, anti-U1RNP $(P=0.008)$, a-Sm $(P=0.027)$, and anti-ribosomal p $(P=0.028)$ significantly negatively correlated with glutathione levels. There has no significant correlation between SLE disease activity indexes (SLEDAI) and levels of C3, C4, and antioxidant enzymes. Conclusions. Erythrocyte superoxide dismutase is significantly lower in both NPSLE and non-NPSLE groups. SLE patients have both higher CRP and autoantibodies level and decreased superoxide dismutase level than the healthy control group.

\section{Introduction}

Autoimmune diseases are characterized by the deviated activation of the innate and adaptive immune systems. Autoantibodies are markers of B-cell activation in adaptive immunity. Some are diagnostic and specific, such as anti-nuclear antibody (ANA) and anti-double stranded deoxyribonucleic acid antibody (a-ds DNA) for systemic lupus erythematosus (SLE) and perinuclear anti-neutrophil cytoplasmic antibodies (p-ANCA) and cytoplasmic anti-neutrophil cytoplasmic antibodies (c-ANCA) for vasculitis.

Inflammation and increased oxidative stress are recognized in almost all patients with autoimmune diseases. The effect of oxidative stress may lead to cell apoptosis and 
further antigen presentation and autoantibody formation. On the other hand, host factor and disease characteristics are important factors $[1,2]$. Lipid peroxidation of erythrocyte membranes by toxic oxygen-free radicals plays a major role in red blood cell hemolysis, which is common in SLE. Increased sensitivity of red blood cells (RBCs) to oxidative damage is therefore an index of antioxidant deficiency. The steadystate formation of oxidants is balanced by a similar rate of their consumption by antioxidants. This study was designed to evaluate changes in the activities of three antioxidant enzymes, including superoxide dismutase (SOD-rbc) and glutathione peroxidase (GPX-rbc) activities on RBCs, and serum glutathione levels of SLE patients.

The SOD-rbc is one of the three forms of SOD in human cytoplasm of cells. Because of the large amount of RBCs widely distributed in blood, SOD in RBCs is a major source of antioxidant against the dismutation of superoxide $\left(\mathrm{O}_{2}{ }^{-}\right)$. The glutathione peroxidase is a group of enzymes that convert lipid hydroperoxides to alcohols and hydrogen peroxide to water and protect organism against oxidative damage. The glutathione is also an antioxidant and concentration of the reduced state of the glutathione as an indicator of antioxidant capacity.

SLE patients may suffer from skin, joint, hematologic, renal, pulmonary, and neurological diseases. Additionally, the course of the disease is highly variable between patients, with certain manifestations more common than others and the overall impact on quality of life dependent on the individual patient's circumstances and particular disease manifestations. Neuropsychiatric symptoms occur in between $30 \%$ and $40 \%$ of SLE patients, which can constitute the initial presentation, and may occur in the context of a SLE flare. The clinical features can experience acute neurological events such as seizures, cerebrovascular accidents, and delirium and psychiatric conditions including depression, anxiety, and psychosis, as well as memory loss and general cognitive decline.

To our knowledge, only several articles have focused specifically on the activities of antioxidant enzymes in peripheral blood of SLE [3-5]. Further, research into the underlying mechanisms of NPSLE and the role of antioxidant reserve was less mentioned elsewhere. This study hypothesizes that antioxidative capacity plays an important role in SLE severity. A better understanding of the pathophysiology of SLE is essential for the treatment or prevention of flareups. This study aimed to determine the relationship among levels of antioxidant enzymes and autoantibodies and severity of SLE.

\section{Patients and Methods}

2.1. Study Patients. This study included 32 SLE patients and 16 healthy controls. The SLE patients were followed up at the Rheumatology Outpatient Clinic between February 2012 and March 2013 and were prospectively evaluated. The diagnostic criteria for SLE were based on the 1997 revised American College of Rheumatology (ACR) classification [6]. The Institutional Review Committee on Human Research approved the study protocol and all participants provided informed consent. Disease activity was recorded according to SLE disease activity index 2000 (SLEDAI-2k) [7]. Characteristically, SLE patients can be classified into two groups, which are NPSLE and non-NPSLE groups, according to the ACR classification [8]. The health subjects, who have not any major underlying diseases or take any medicines including antioxidant agents, were enrolled as control group.

2.2. Blood Sampling and Assessment of Oxidative Stress. Blood samples were collected by venipuncture of forearm veins from the patients and controls.

\subsection{Erythrocyte Assay of Antioxidant Enzymes}

2.3.1. Erythrocyte Superoxide Dismutase (SOD) Activity. Superoxide dismutase activity was measured in erythrocytes using a commercially available kit (Ransod, Randox Lab, Grumlin, UK) based on the method developed by McCord and Fridovich [9]. The role of SOD was to accelerate the dismutation produced during oxidative energy processes of superoxide radicals $\left(\mathrm{O}_{2}{ }^{-}\right)$to hydrogen peroxide and molecular oxygen. This method employed xanthine and xanthine oxidase to generate superoxide radicals that react with 2-(4-iodophenyl)-3-(4-nitrophenol)-5phenyltetrazolium chloride to form a red formazan dye. The SOD activity was then measured by the degree of inhibition of this reaction. The assay was conducted on washed RBCs by diluting the samples to obtain $30 \%-60 \%$ inhibition. A standard was supplied with the kit and was diluted to provide a range of standards and a calibration curve. A standard curve was produced by plotting the percentage (\%) inhibition for each standard against $\log 10$. The result was multiplied by the appropriate dilution factor (100) and expressed in units/liter (U/L) of whole blood.

2.3.2. Erythrocyte Glutathione Peroxidase (GPX) Activity. Erythrocyte GPX activity was measured using a commercially available kit (Ransel; Randox Lab, Crumlin, UK). Heparinized whole blood samples were diluted to convert glutathione peroxidase to its reduced form. The sample was incubated for $5 \mathrm{~min}$ and then diluted with Drabkin's reagent to avoid false elevation due to the presence of peroxidases in human blood. The diluted sample was mixed with reagent (glutathione, glutathione reductase, and NADPH) and cumene hydroperoxide. Glutathione (GSH) was reduced by cumene hydroperoxide and then oxidized in a GPXcatalyzed reaction. In the presence of glutathione reductase (GR) and NADPH, the oxidized glutathione (GSSG) was immediately converted to the reduced form with a concomitant oxidation of NADPH to NADP+. The decrease in absorbance after 1 and $2 \mathrm{~min}$ at $340 \mathrm{~nm}$ was measured. The results were expressed in units/liter of hemolysate and multiplied by the appropriate dilution factor to obtain result in $\mathrm{U} / \mathrm{L}$ of whole blood.

2.3.3. Determination of Serum Glutathione Content. The ability of antioxidative defense in response to increased oxidative damage was evaluated by measuring the plasma 
level of total reduced glutathione as plasma glutathione physiologically free of radical scavengers. Plasma free glutathione was determined by directly reacting glutathione with 5,5-dithiobis 2-nitrobenzoic acid (DTNB) to form 5-thio2-nitrobenzoic acid (TNB). The amount of glutathione in the sample was calculated from the absorbance using the extinction coefficient of TNB (molar extinction coefficient of TNB was $13,600 \mathrm{M}^{-1} \mathrm{~cm}^{-1}$ at $412 \mathrm{~nm}$ ).

2.4. Autoantibody Detection. Autoantibody titers, including IgG antibodies against dsDNA (a-dsdna), Ribosomal p (arib p), Ro (52/60) (a-ro), Ro $52 \mathrm{kDa}$ (a-ro52), Ro $60 \mathrm{kDa}$ (a-ro60), La (a-la), U1RNP (a-ulrnp), Sm (a-sm), Mi-2 (a$\mathrm{mi2}$ ), perinuclear anti-neutrophil cytoplasmic antibodies (pANCA), cytoplasmic anti-neutrophil cytoplasmic antibodies (c-ANCA), and Jo-1 (a-jol), were detect by Thermo Scientific Phadia system. They were detected by the system according to the operations manual.

2.5. Statistical Analysis. Data were expressed as mean \pm SD or median (interquartile range). Categorical variables were compared using Chi-square test or Fisher's exact test, as appropriate. Levels of continuous variables were logarithmically transformed to improve normality. Univariate analyses were compared using Student's $t$-test. Continuous variables among three groups (NPSLE and non-NPSLE and control groups) were compared using one-way ANOVA, followed by post hoc multiple comparison procedure. Correlation analysis was used to evaluate the relationship between level of autoantibodies and oxidative stress markers using the Spearman method. Statistical significance was set at $P<$ 0.05 . All statistical calculations were performed using the SAS software package, version 9.1 (2002, SAS Statistical Institute, North Carolina).

\section{Results}

3.1. Baseline Characteristics of the Study Patients. Thirtytwo adults aged 26-58 years with SLE were enrolled. The baseline clinical characteristics and laboratory data of the patient and control groups were listed in Tables 1 and 2. There was no significant difference in age and sex. The clinical symptoms of the 32 SLE patients included neurological involvement in 15, musculoskeletal involvement in 12 , renal involvement in 10, mucocutaneous involvement in five, hematologic involvement in four, vasculitis in four, and cardiorespiratory involvement in two patients. Fifteen patients with neurological involvement were classified as NPSLE, and the other 17 patients were non-NPSLE group. Their available clinical autoantibody titers and three oxidative stress markers, including glutathione, SOD-rbc, and GPX$\mathrm{rbc}$, were also listed. The results demonstrated that CRP levels are higher in non-NPSLE group as compared with control group $(P=0.034)$. The CRP levels were not significantly different between NP-SLE and non-NP-SLE groups $(P=$ 0.997). The SOD-rbc levels are significantly lower in both NPSLE and non-NPSLE groups, as compared with control group (both $P$ value $<0.001$ ) (Table 1).
3.2. Correlation among Oxidative Stress Markers, Antibodies, and Diseases Severity Scores. There was no significant correlation between SLE disease activity indexes (SLEDAI) and levels of C3, C4, and antioxidant enzymes. Autoantibody titers, including a-ulrnp $(P=0.008)$, a-Sm $(P=0.027)$, and a-rib p $(P=0.028)$, significantly but negatively correlated with serum glutathione level (Table 3 ).

\section{Discussion}

Oxidative stress represents the sum of inflammation in patients, including subjective [10] and objective [11] measures. Several lines of evidence reveal that poor clinical outcome is correlated with elevated oxidative stress. Outcome measurement ranges from high-dose immunosuppressants $[12,13]$, prolonged steroid treatment [14], early atherosclerosis [15], insulin-resistance [16, 17], hypertension [18], proteinuria [19], and liver damage [20] to overall systemic lupus erythematosus disease activity index [21]. Previous studies have focused on antiphospholipid and autoantibodies [22, 23], whereas the present one shows another link between autoantibodies and oxidative stress.

This study has two major findings. First, superoxide dismutase in RBC is significantly lower in both NPSLE and non-NPSLE as compared with the control group. Second, the autoantibodies, including a-ulrnp $(P=0.008)$, a-Sm $(P=0.027)$, and a-rib $\mathrm{p}(P=0.028)$, significantly negatively correlate with serum glutathione level. Clinically, these autoantibodies represent different patient subsets. For instance, a-ulrnp is a marker for mixed connective tissue disease and is associated with mild forms of SLE [24]. a-Sm and a-rib p are specific for SLE [25], while an a-rib p has been proposed as a marker of NPSLE [26].

There is a trend of lower GPx-rbc in SLE patients, but not reach statistically significance. This might be due to the fact that the clinical symptoms of the SLE patients vary from mild disease (represented by positive a-ulrnp) to neuropsychiatric involvement (represented by a-rib $\mathrm{p}$ ). The disease pathogenesis is also heterogeneous in SLE patients; as a result, antioxidant enzyme in RBC may not be sufficient to represent the overall oxidative stress. Compartmentalized oxidative stress in either self-reactive $\mathrm{T}$ cells, B cells, or phagocytic cells should be further studied [13].

NPSLE is a more severe form and may be a devastating subgroup of SLE, compared to non-NPSLE [27, 28]. In our study, we show that NPSLE and non-NPSLE patients have similar antioxidant reserves and CRP levels. This might indicate that the pathogenesis of NPSLE might not depend on the traditional inflammatory pathway, which cannot be reflected on the antioxidant reserves and CRP levels or just because these patients were in the convalescent stage. The pathogenesis of NPSLE could be either antibody dependent $[29,30]$ or degeneration [31-33], which both show little inflammation. The SLE disease activities were not different between NP-SLE and non-NPSLE in our study, which might be due to selection bias. We collected our patients from outpatient clinic, who are mostly stabilized patients. 


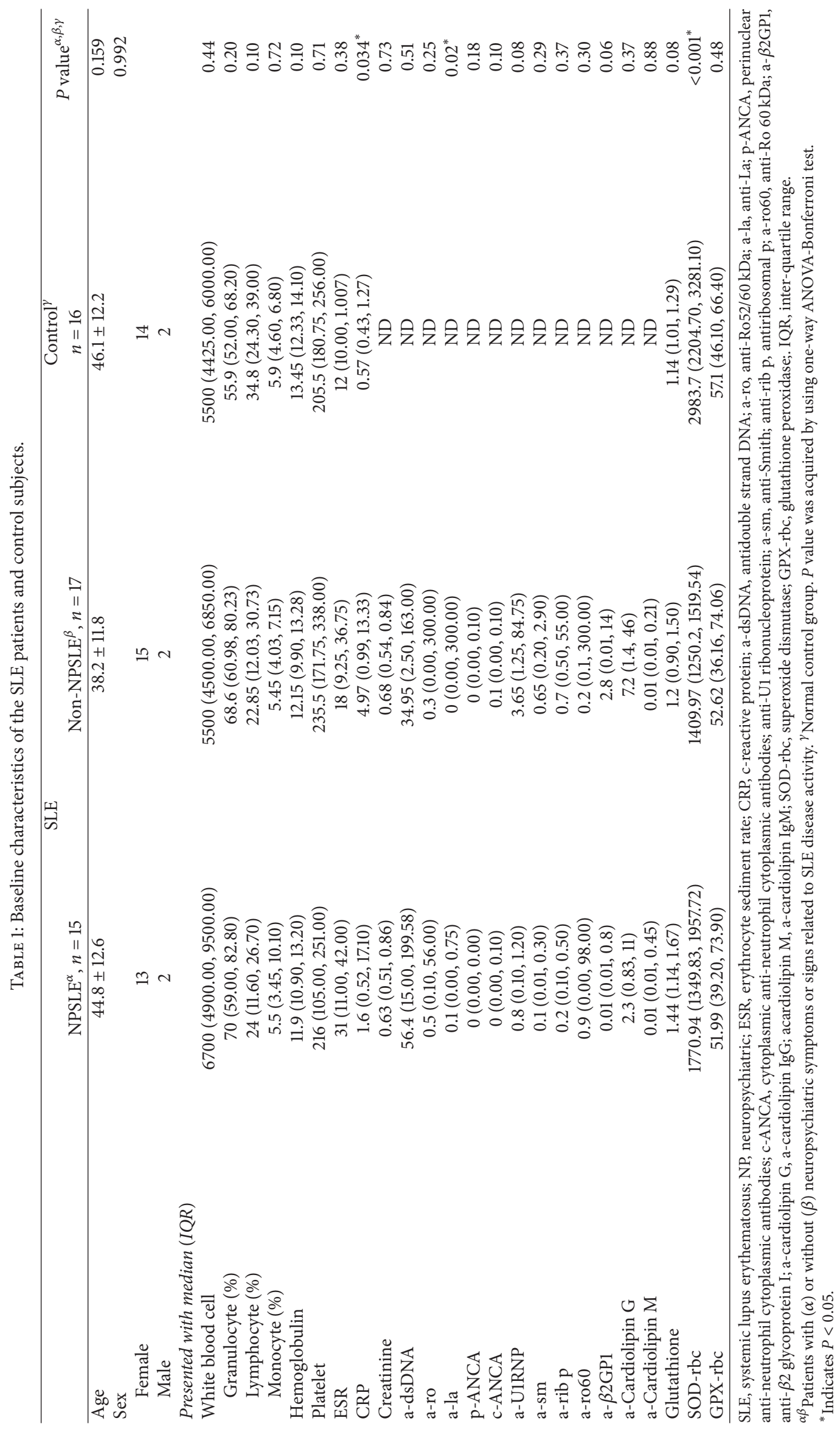


TABLE 2: Clinical data of SLE patients.

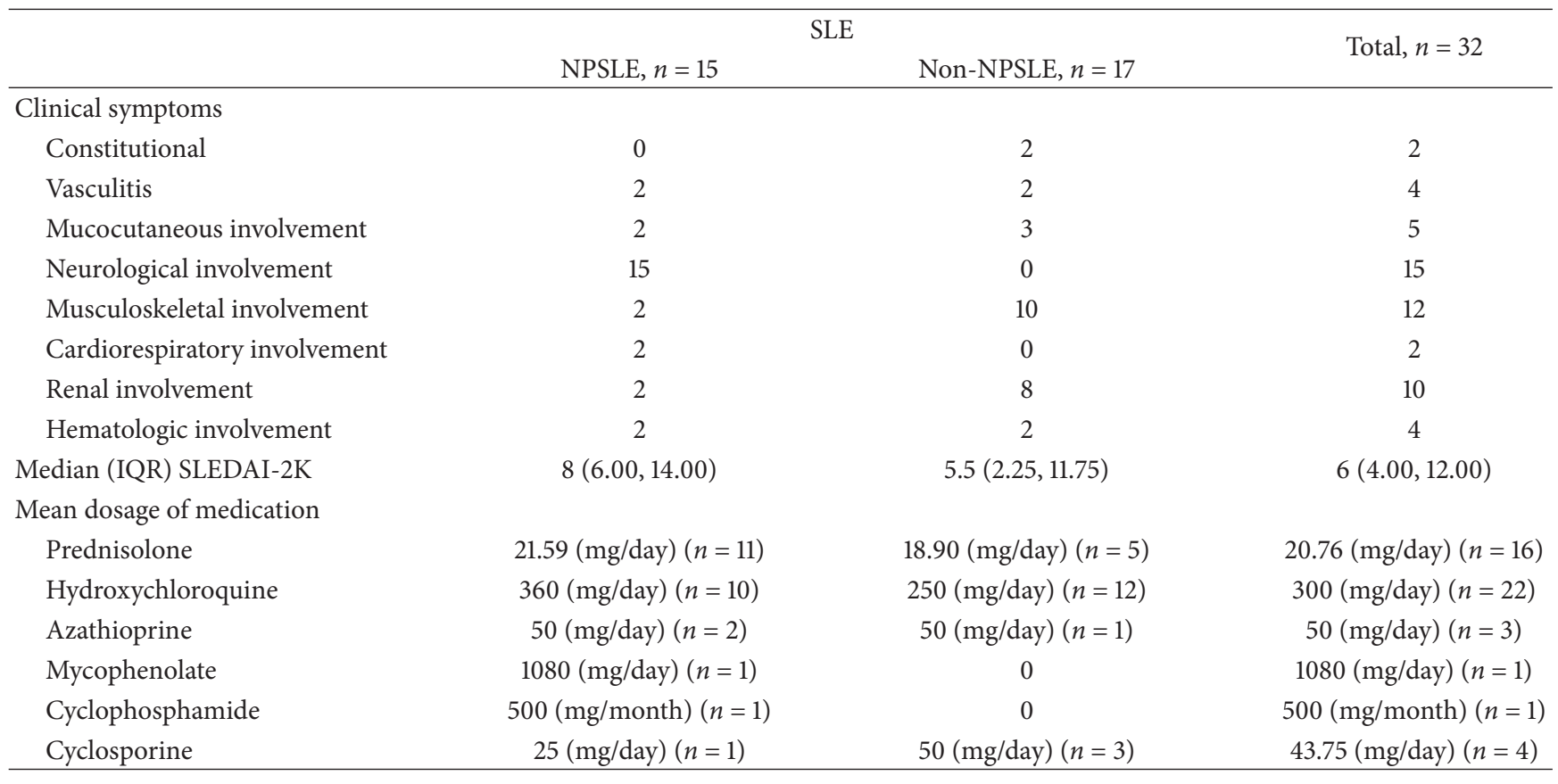

TABLE 3: Correlation among oxidative stress markers, antibodies, and diseases severity scores in SLE.

\begin{tabular}{|c|c|c|c|c|}
\hline \multirow{2}{*}{ Variables } & & \multicolumn{3}{|c|}{ Biomarker of antioxidants } \\
\hline & & Glutathione & SOD-rbc & GPX-rbc \\
\hline \multirow{2}{*}{ SLEDAI } & Correlation coefficient & -0.295 & 0.017 & 0.123 \\
\hline & $P$ value & 0.113 & 0.929 & 0.519 \\
\hline \multirow{2}{*}{ C3 } & Correlation coefficient & 0.177 & 0.086 & 0.083 \\
\hline & $P$ value & 0.35 & 0.651 & 0.663 \\
\hline \multirow{2}{*}{$\mathrm{C} 4$} & Correlation coefficient & -0.08 & 0.073 & 0.008 \\
\hline & $P$ value & 0.673 & 0.701 & 0.968 \\
\hline \multirow{2}{*}{ a-dsDNA } & Correlation coefficient & 0.02 & 0.119 & 0.247 \\
\hline & $P$ value & 0.917 & 0.54 & 0.197 \\
\hline \multirow{2}{*}{ a-U1RNP } & Correlation coefficient & $-0.588^{* *}$ & -0.451 & -0.409 \\
\hline & $P$ value & 0.008 & 0.053 & 0.147 \\
\hline \multirow{2}{*}{ a-Sm } & Correlation coefficient & $-0.506^{*}$ & -0.269 & -0.291 \\
\hline & $P$ value & 0.027 & 0.265 & 0.314 \\
\hline \multirow{2}{*}{ a-rib p } & Correlation coefficient & $-0.490^{*}$ & -0.348 & 0.184 \\
\hline & $P$ value & 0.028 & 0.132 & 0.437 \\
\hline \multirow{2}{*}{ CRP } & Correlation coefficient & 0.236 & -0.315 & 0.191 \\
\hline & $P$ value & 0.268 & 0.134 & 0.371 \\
\hline
\end{tabular}

${ }^{*} P<0.05 ;{ }^{* *} P<0.01$.

$r$, correlation coefficient; SLEDAI, systemic lupus erythematosus disease activity index; C3, complement 3; C4 complement 4; a-dsDNA, anti-double strand DNA; a-rib p, antiribosomal p; a-U1RNP, anti-U1 ribonucleoprotein; CRP, c-reactive protein; SOD-rbc, superoxide dismutase activity on red blood cell; GPX$\mathrm{rbc}$, glutathione peroxidase activity on red blood cell

The a-ulrnp $(P=0.019)$ was the only autoantibody that significantly negatively correlated with glutathione peroxidase activity on red blood cell (GPX-rbc) among neuropsychiatric SLE subgroups.

This study also has several limitations. First, most of the SLE patients were in the convalescent stage. Thus, there was no significant correlation between SLEDAI and C3, C4, and antioxidant enzyme levels. Second, this is a cross-sectional observational study. Several immunosuppressant drugs (e.g., steroids) or antioxidant agents (e.g., vitamin E, coenzyme Q10) could influence the levels of antioxidant enzymes and may cause potential bias in the statistical analysis. Third, the diversity of SLE patients and various disease pathogenesis could be a bias in such a cross-sectional study. Lastly, 
the case numbers are small. Large-scale prospective studies are warranted to evaluate the prognostic contribution of leukocyte apoptosis on clinical outcomes.

In conclusion, erythrocyte superoxide dismutase is significantly lower in both NPSLE and non-NPSLE groups. Among the autoantibodies, a-ulrnp, a-Sm, and a-rib p are significantly correlated with serum glutathione levels. Further understanding of the specific mechanisms of antioxidative capacity in SLE may lead to new strategies that will improve patient outcome.

\section{Ethical Approval}

This study was approved by Chang Gung Memorial Hospital's Institutional Review Committee on Human Research.

\section{Conflict of Interests}

The authors declare that they have no conflict of interests.

\section{Acknowledgments}

This work was supported by grants from Chang Gung Memorial Hospital (Chang Gung Medical Research Project CMRPG870991 and NHRI-EX101-10142EI). The authors wish to thank Dr. Gene Alzona Nisperos for editing and reviewing the paper for English language considerations.

\section{References}

[1] W. Ollier, E. Davies, N. Snowden et al., "Association of homozygosity for glutathione-S-transferase GSTM1 null alleles with the Ro+/La- autoantibody profile in patients with systemic lupus erythematosus," Arthritis and Rheumatism, vol. 39, no. 10, pp. 1763-1764, 1996.

[2] C. Kiyohara, M. Washio, T. Horiuchi et al., "Risk modification by CYP1A1 and GSTM1 polymorphisms in the association of cigarette smoking and systemic lupus erythematosus in a Japanese population," Scandinavian Journal of Rheumatology, vol. 41, no. 2, pp. 103-109, 2012.

[3] I. K. Mohan and U. N. Das, "Oxidant stress, anti-oxidants and essential fatty acids in systemic lupus erythematosus," Prostaglandins Leukotrienes and Essential Fatty Acids, vol. 56, no. 3, pp. 193-198, 1997.

[4] P. E. Morgan, A. D. Sturgess, and M. J. Davies, "Increased levels of serum protein oxidation and correlation with disease activity in systemic lupus erythematosus," Arthritis and Rheumatism, vol. 52, no. 7, pp. 2069-2079, 2005.

[5] M. D. Evans, M. S. Cooke, M. Akil, A. Samanta, and J. Lunec, "Aberrant processing of oxidative DNA damage in systemic lupus erythematosus," Biochemical and Biophysical Research Communications, vol. 273, no. 3, pp. 894-898, 2000.

[6] E. L. Smith and R. H. Shmerling, "The American college of rheumatology criteria for the classification of systemic lupus erythematosus: strengths, weaknesses, and opportunities for improvement," Lupus, vol. 8, no. 8, pp. 586-595, 1999.

[7] D. D. Gladman, D. Ibañez, and M. B. Urowltz, "Systemic lupus erythematosus disease activity index 2000," Journal of Rheumatology, vol. 29, no. 2, pp. 288-291, 2002.
[8] J. G. Hanly, "ACR classification criteria for systemic lupus erythematosus: limitations and revisions to neuropsychiatric variables," Lupus, vol. 13, no. 11, pp. 861-864, 2004.

[9] J. M. McCord and I. Fridovich, "Superoxide dismutase. An enzymic function for erythrocuprein (hemocuprein)," The Journal of Biological Chemistry, vol. 244, no. 22, pp. 6049-6055, 1969.

[10] I. Avalos, C. P. Chung, A. Oeser et al., "Oxidative stress in systemic lupus erythematosus: relationship to disease activity and symptoms," Lupus, vol. 16, no. 3, pp. 195-200, 2007.

[11] P. R. J. Ames, J. Alves, I. Murat, D. A. Isenberg, and J. NouroozZadeh, "Oxidative stress in systemic lupus erythematosus and allied conditions with vascular involvement," Rheumatology, vol. 38, no. 6, pp. 529-534, 1999.

[12] P. Kovacic and J. D. Jacintho, "Systemic lupus erythematosus and other autoimmune diseases from endogenous and exogenous agents: unifying theme of oxidative stress," Mini Reviews in Medicinal Chemistry, vol. 3, no. 6, pp. 568-575, 2003.

[13] A. Perl, "Oxidative stress in the pathology and treatment of systemic lupus erythematosus," Nature Reviews Rheumatology, vol. 9, no. 674, p. 686, 2013.

[14] J. D. Alves and P. R. J. Ames, "Atherosclerosis, oxidative stress and auto-antibodies in systemic lupus erythematosus and primary antiphospholipid syndrome," Immunobiology, vol. 207, no. 1, pp. 23-28, 2003.

[15] E. Matsuura, K. Kobayashi, and L. R. Lopez, "Atherosclerosis in autoimmune diseases," Current Rheumatology Reports, vol. 11, no. 1, pp. 61-69, 2009.

[16] M. Lozovoy, A. Simao, S. Oliveira et al., "Relationship between iron metabolism, oxidative stress, and insulin resistance in patients with systemic lupus erythematosus," Scandinavian Journal of Rheumatology, vol. 42, no. 4, pp. 303-310, 2013.

[17] S. S. Koca, I. Karaca, M. F. Yavuzkir et al., "Insulin resistance is related with oxidative stress in systemic lupus erythematosus," The Anatolian Journal of Cardiology, vol. 9, no. 1, pp. 23-28, 2009.

[18] M. J. Ryan, "The pathophysiology of hypertension in systemic lupus erythematosus," The American Journal of PhysiologyRegulatory Integrative and Comparative Physiology, vol. 296, no. 4, pp. R1258-R1267, 2009.

[19] K. W. Mathis, M. Venegas-Pont, C. W. Masterson, N. J. Stewart, K. L. Wasson, and M. J. Ryan, "Oxidative stress promotes hypertension and albuminuria during the autoimmune disease systemic lupus erythematosus," Hypertension, vol. 59, no. 3, pp. 673-679, 2012.

[20] M. Lozovoy, A. Simão, C. Panis et al., "Oxidative stress is associated with liver damage, inflammatory status, and corticosteroid therapy in patients with systemic lupus erythematosus," Lupus, vol. 20, no. 12, pp. 1250-1259, 2011.

[21] K. Tewthanom, S. Janwityanuchit, K. Totemchockchyakarn, and D. Panomvana, "Correlation of lipid peroxidation and glutathione levels with severity of systemic lupus erythematosus: a pilot study from single center," Journal of Pharmacy and Pharmaceutical Sciences, vol. 11, no. 3, pp. 30-34, 2008.

[22] F. I. Romero, M. A. Khamashta, and G. R. V. Hughes, "Lipoprotein(a) oxidation and autoantibodies: a new path in atherothrombosis," Lupus, vol. 9, no. 3, pp. 206-209, 2000.

[23] E. Matsuura, K. Kobayashi, T. Yasuda, and T. Koike, "Antiphospholipid antibodies and atherosclerosis," Lupus, vol. 7, supplement 2, pp. S135-S139, 1998.

[24] T. Ilić, S. Curić, S. Vodopivec et al., "Anti-U1RNP antibodies and lupus nephritis," Medicinski Pregled, vol. 53, no. 11-12, pp. 589594,2000 . 
[25] D. Carmona-Fernandes, M. J. Santos, H. Canhao, and J. E. Fonseca, "Anti-ribosomal P protein IgG autoantibodies in patients with systemic lupus erythematosus: diagnostic performance and clinical profile," BMC Medicine, vol. 11, article 98, 2013.

[26] S. Madrane and C. Ribi, "Central neuropsychiatric involvement in systemic lupus erythematosus," Revue Médicale Suisse, vol. 8, no. 337, pp. 848-853, 2012.

[27] G. K. Bertsias, J. P. A. Ioannidis, M. Aringer et al., "EULAR recommendations for the management of systemic lupus erythematosus with neuropsychiatric manifestations: report of a task force of the EULAR standing committee for clinical affairs," Annals of the Rheumatic Diseases, vol. 69, no. 12, pp. 2074-2082, 2010.

[28] M. Govoni, S. Bombardieri, A. Bortoluzzi et al., "Factors and comorbidities associated with first neuropsychiatric event in systemic lupus erythematosus: does a risk profile exist? A large multicentre retrospective cross-sectional study on 959 italian patients," Rheumatology, vol. 51, no. 1, Article ID ker310, pp. 157168, 2012.

[29] R. M. Pereira, N. H. Yoshinari, R. M. de Oliveira, and W. Cossermelli, "Antiganglioside antibodies in patients with neuropsychiatric systemic lupus erythematosus," Lupus, vol. 1, no. 3, pp. 175-179, 1992.

[30] J. G. Hanly, M. B. Urowitz, L. Su et al., "Autoantibodies as biomarkers for the prediction of neuropsychiatric events in systemic lupus erythematosus," Annals of the Rheumatic Diseases, vol. 70, no. 10, pp. 1726-1732, 2011.

[31] Y. Yoshikawa, H. Mizutani, and M. Shimizu, "Systemic lupus erythematosus with ischemic peripheral neuropathy and lupus anticoagulant: response to intravenous prostaglandin El," Cutis, vol. 58, no. 6, pp. 393-396, 1996.

[32] Y. P. Guo, "Sensorimotor and autonomic neuropathy associated with systemic lupus erythematosus," Chinese Journal of Neurology and Psychiatry, vol. 22, no. 3, pp. 163-191, 1989.

[33] P. A. McCombe, J. G. McLeod, J. D. Pollard, Y. P. Guo, and T. J. Ingall, "Peripheral sensorimotor and autonomic neuropathy associated with systemic lupus erythematosus. Clinical, pathological and immunological features," Brain, vol. 110, part 2, pp. 533-549, 1987. 

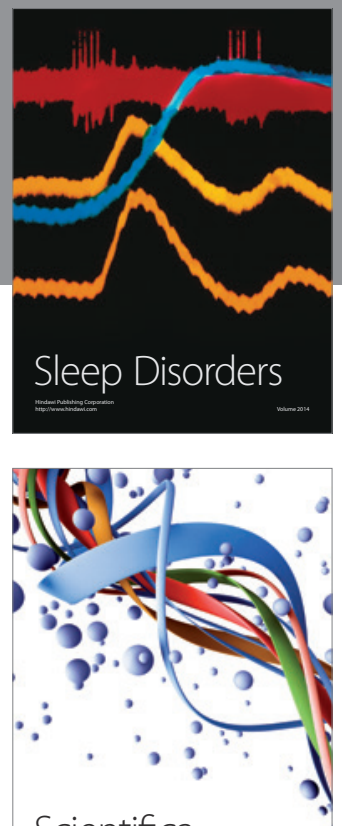

Scientifica
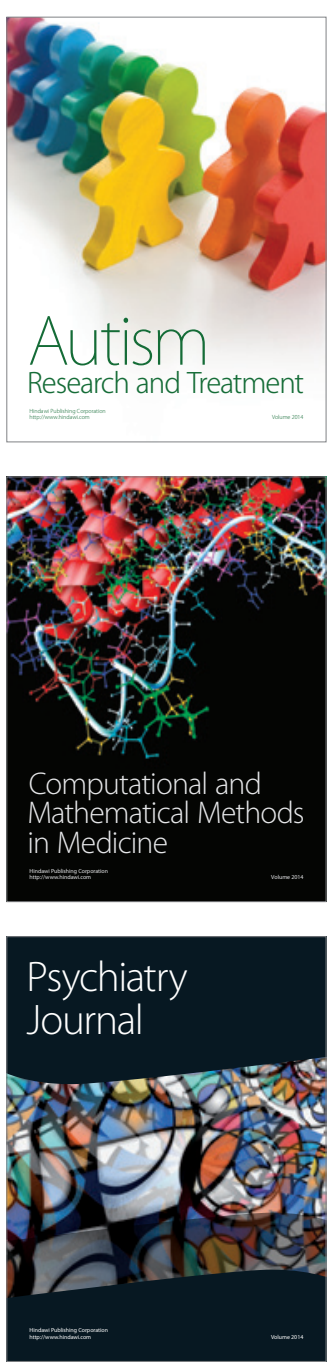
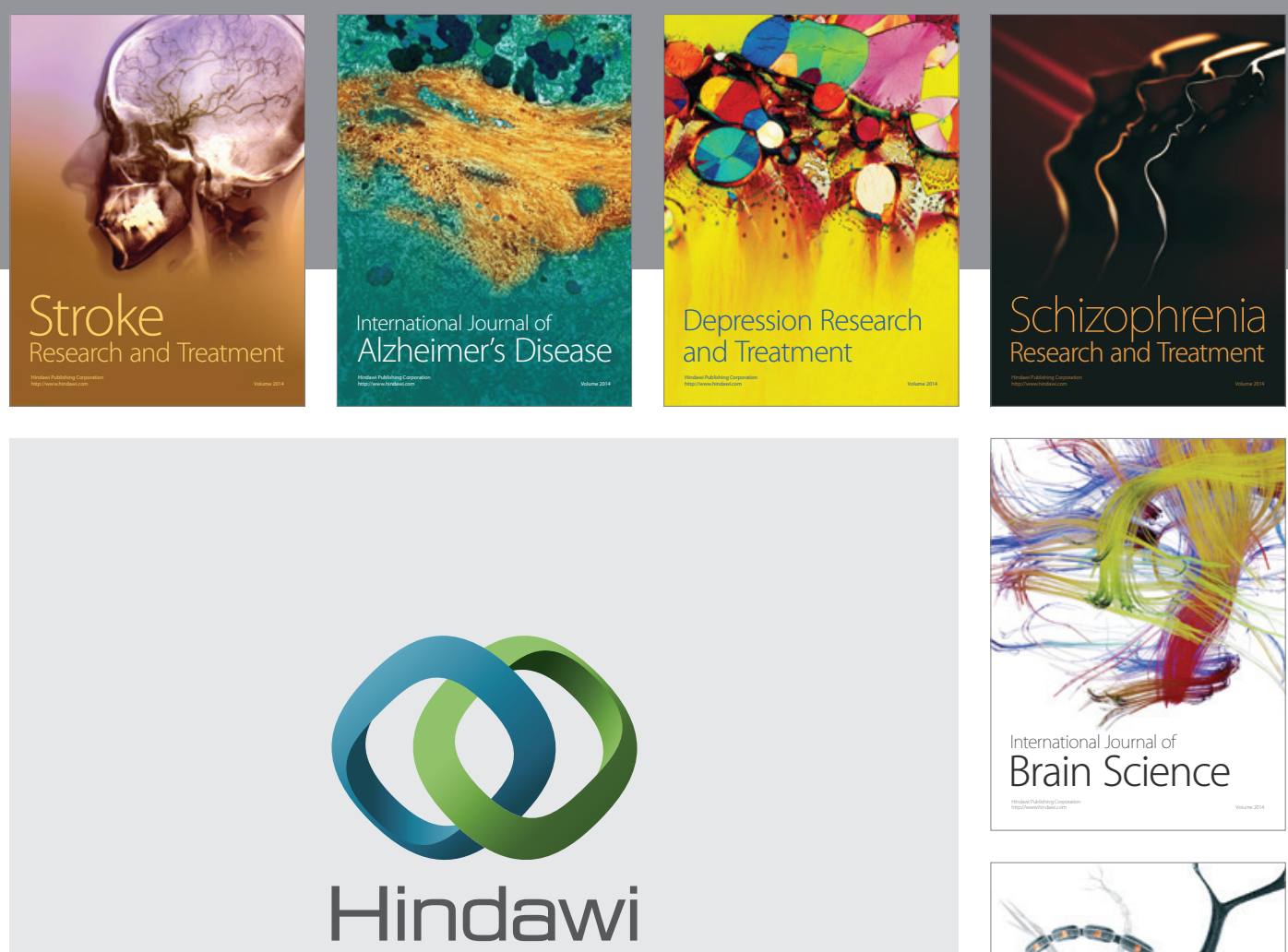

Submit your manuscripts at

http://www.hindawi.com
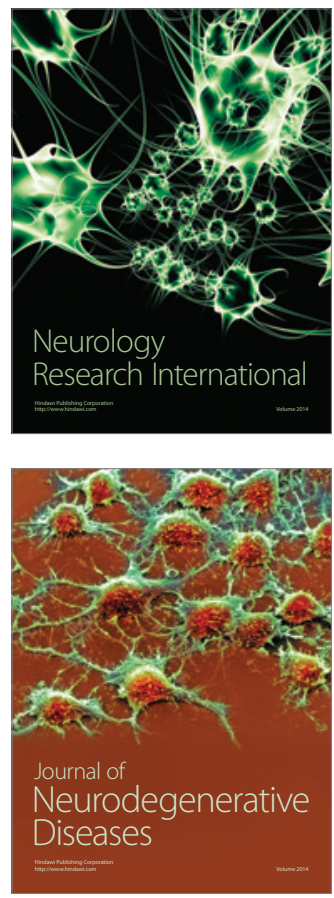

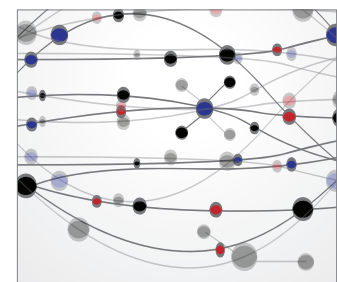

The Scientific World Journal
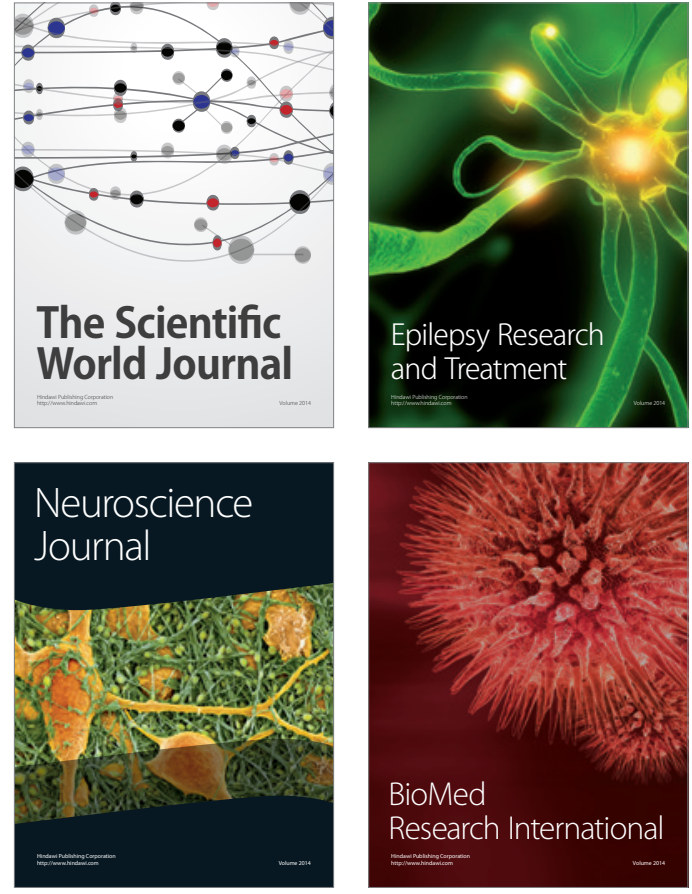

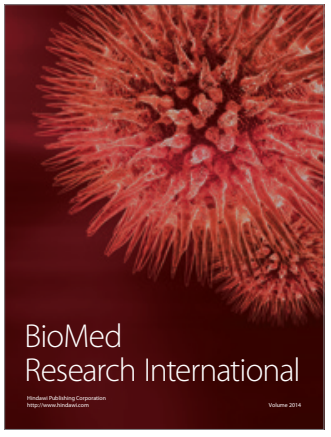

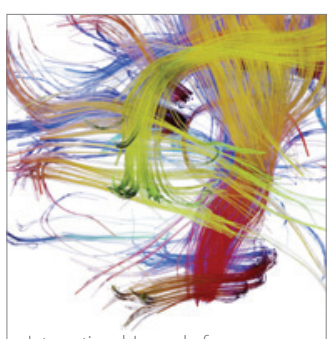

Brain Science

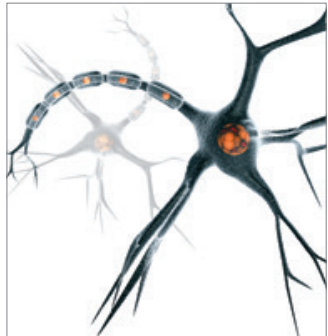

Neural Plasticity
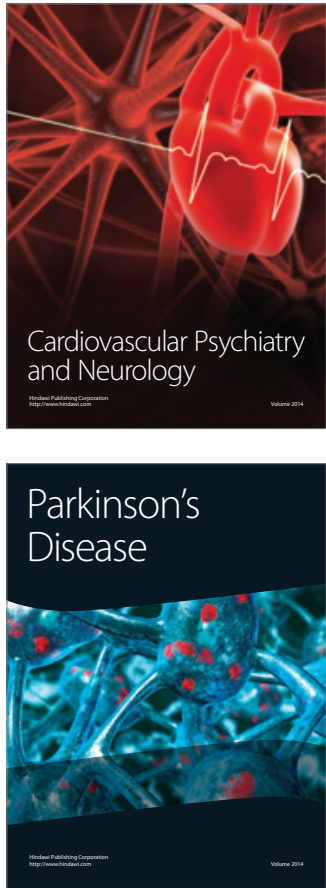